\title{
Near-infrared sky background fluctuations at mid- and low latitudes
}

\author{
G. Moreels • J. Clairemidi • M. Faivre • D. Pautet • \\ F. Rubio da Costa $•$ P. Rousselot • J.W. Meriwether • \\ G.A. Lehmacher • E. Vidal • J.L. Chau • G. Monnet
}

Received: 9 September 2007 / Accepted: 21 March 2008 /

Published online: 15 May 2008

(C) The Author(s) 2008

\begin{abstract}
The emission of the upper atmosphere introduces an additional variable component into observations of astronomical objects in the NIR 700-3,000 nm range. The subtraction of this component is not easy because it varies during the night by as much as $100 \%$ and it is not homogeneous over the sky. A program aimed at measuring and understanding the main characteristics of the atmospheric NIR emission was undertaken. A $512 \times 512$ CCD camera equipped with a RG780/2 mm filter is used to obtain images of the sky in a $36^{\circ} \times 36^{\circ}$ field of view. The intensities of a given star and of the nearby region devoid of star in a $439 \operatorname{arcmin}^{2}$ area are monitored during periods of time of several hours. The sky intensity measured in the 754-900 $\mathrm{nm}$ bandpass, reduced to zenith and zero airmass is comprised between mag20 and mag18.5 per arcsecond ${ }^{2}$. A diminution by a factor of two during the night is frequently observed. Intensity fluctuations having an amplitude of $15 \%$ and periods of 5-40 min are present in the images with a structure of regularly spaced stripes. The fluctuations of the NIR sky background intensity are due to (1) the chemical evolution of the upper atmosphere composition during the night and (2)
\end{abstract}

\footnotetext{
G. Moreels $(\bowtie) \cdot$ J. Clairemidi $\cdot$ D. Pautet $\cdot$ F. Rubio da Costa $\cdot$ P. Rousselot Observatoire de Besançon, Institut Utinam, CNRS-Université de Franche Comté UMR 6213, BP 1615, 25010 Besançon, France

e-mail: guy.moreels@obs-besancon.fr

M. Faivre $\cdot$ J. Meriwether $\cdot$ G. Lehmacher

Department of Physics and Astronomy, Clemson University, Clemson, SC 29634-0978, USA

F. Rubio da Costa

Universidad de La Laguna, 38203 Tenerife, Spain

E. Vidal $\cdot$ J. Chau

Instituto Geofísico del Perú, Calle Badajoz 169, Mayorazgo IV Etapa, Lima, Perú

G. Monnet

ESO, Karl-Schwarzschild-Str. 2, D-85748 Garching bei München, Germany
} 
dynamical processes such as tides with periods of 3-6 h or gravity waves with periods of several tens of minutes. We suggest that a monitoring of the sky background intensity could be set up when quantitative observations of astronomical objects require exposure times longer than $\sim 10 \mathrm{~min}$. The publication is illustrated with several video films accessible on the web site http://www.obs-besancon.fr/ nirsky/. Enter username: nirsky and password: skynir.

Keywords Atmospheric effects · Sky background - Image processing · Infrared · Stars

\section{Introduction}

If the human eye were sensitive to radiation in the near-infrared range, at wavelengths slightly longer than $700 \mathrm{~nm}$, its perception of a perfectly cloudless night sky would drastically differ from the usual view that we have of a profound star-spangled hemispheric cup (Figs. 1 and 2). The sky would appear as a wide striped field, the lines having an overall direction frequently oriented from the $\mathrm{W}-$ SW to the E-SE azimuth vanishing points in the horizon. The observed emission is due to chemiluminescent processes occurring in the upper atmosphere in a region called mesosphere that corresponds to a minimum in the vertical temperature profile. The atmospheric emission in the near-infrared was initially detected by Meinel in 1950 [1] when fast grating spectrographs became available. It was identified as being due to the $\mathrm{O}_{2}$ and $\mathrm{OH}$ molecules produced as excited species in specific chemical processes occurring under low pressure conditions [2]. The $\mathrm{OH}^{*}$ molecules are produced in the reaction of atomic hydrogen with ozone [3]. They emit a series of rotation-vibration bands that cover an extended part of the spectrum from $650 \mathrm{~nm}$ to $3 \mu \mathrm{m}$ [4-6]. This emission is quenched if collisions happen with a time-constant shorter than the radiative lifetime. When near-infrared sensitive photographic films became available, images of the atmospheric emission could be obtained with exposure times of 5-10 min [7-10]. The images exhibited structures which showed that the $\mathrm{OH}$ emission is an excellent tracer of the mesospheric dynamical processes [11], i.e. turbulence, tides and gravity waves [12]. Atmospheric gravity waves can be generated in a stably stratified atmosphere placed in a gravity field. They are initiated by perturbations creating harmonic oscillations of fluid particles submitted to gravity acting as a restoring force. They propagate in vertical, horizontal or oblique directions and transport mechanical energy.

Astronomical observation from a ground-based site implies serious difficulties due to presence of the atmosphere. The first problem results from the atmospheric turbulence that is omnipresent in the tropospheric layer $[13,14]$. The turbulence level is usually measured by the seeing parameter which can be higher than $1 \operatorname{arcsec}$ [15].

A second difficulty is due to the sky background which, in the visible, mainly results from the radiation scattered by the lower atmosphere. In the near-infrared, an additional source of radiation is the nightglow, i.e. the luminescence of the atmosphere itself. With the introduction of infrared focal-plane arrays, astronomical observations are now frequently located in the infrared part of the spectrum. Several survey programs such as DENIS [16], 2MASS [17], TCS-CAIN [18] were initiated. These programs aim at elaborating large photometric data bases in the R, I, J and $\mathrm{K}$ 
Fig. 1 Image of the sky taken during the night of 18 to 19 April 2007 at 01:16 UT. A visible BG18 filter was used. Observation site: Reugney (Doubs, 46 59' 53"N, 6 $8^{\prime} 49^{\prime \prime}$ )

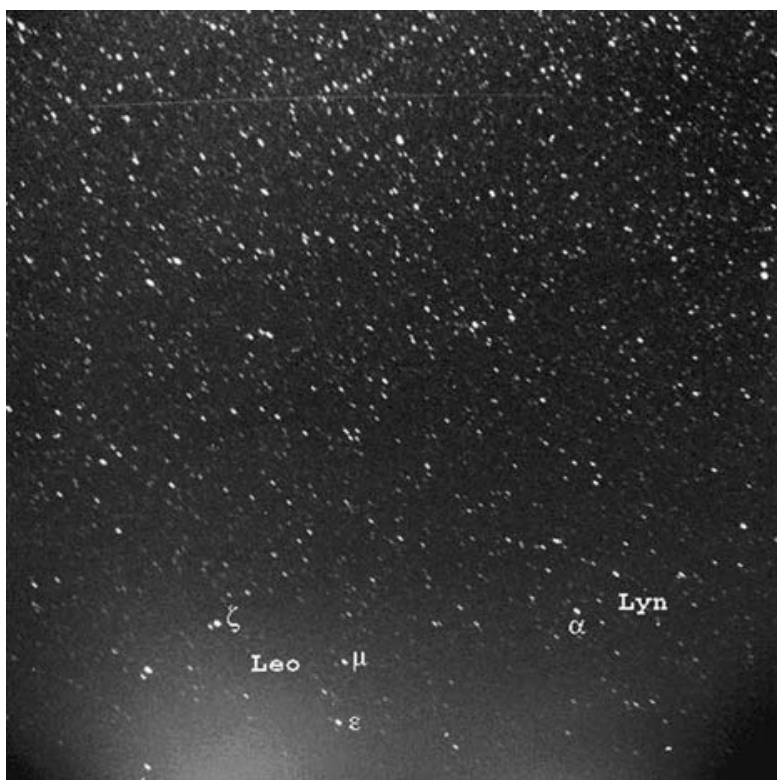

red and near-infrared filters. More recently, the initial stages in implementing VISTA (Visible and Infrared Survey Telescope for Astronomy) began at the Paranal Observatory. This $4 \mathrm{~m}$ telescope will perform extensive surveys of the southern skies in the $0.85-2.3$ micrometer spectral region $[19,20]$. Its field of view will be as large as $1.5^{\circ} \times 1.0^{\circ}$ in order to rapidly cover large areas of the sky and produce monitoring of

Fig. 2 Image of the same part of the sky as in Fig. 1 taken in the near-infrared $5 \mathrm{~min}$ before the visible image. An RG780 filter was used. The apparent structures are due to the $\mathrm{OH}$ emission originating from the mesospheric emissive layer at $\sim 87 \mathrm{~km}$ altitude

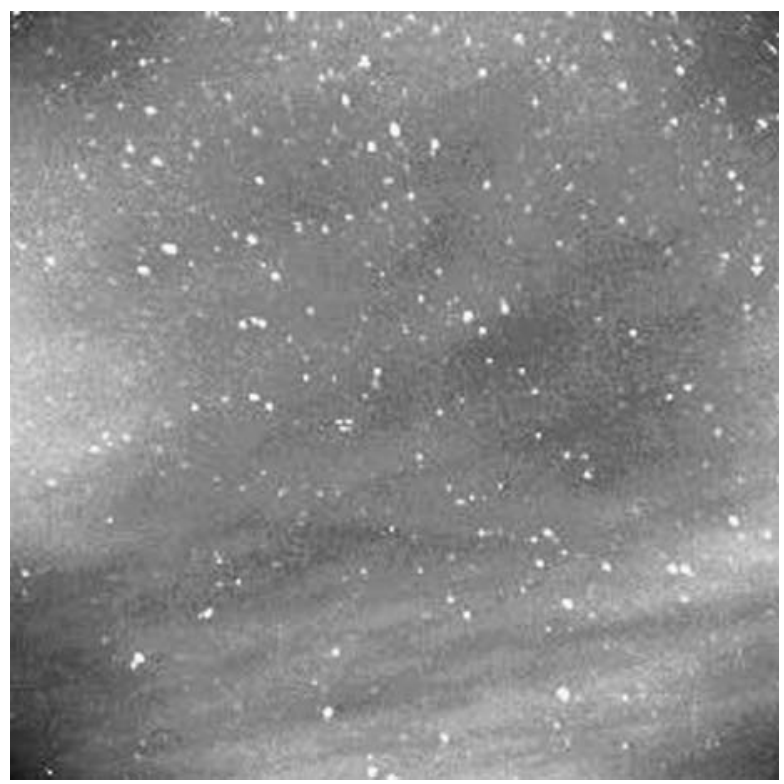


selected objects. In addition, observation of distant objects such as quasars or radiogalaxies which show a high redshift due to Doppler effect results in a displacement of the Balmer series from the UV-visible to the visible-near-infrared region. For example, the $656.3 \mathrm{~nm} \mathrm{H}_{\alpha}$ line for an object at a distance characterized by the Doppler parameter $z=\Delta \lambda / \lambda=0.7$ appears for a terrestrial observer at $\lambda=1,116 \mathrm{~nm}$.

In some fields in astrophysics, programs aimed at determining fundamental parameters rely on the precision of the magnitudes and color indexes of the observed objects. For instance, the variance of the Surface Brightness Fluctuations (SBF) related to certain types of galaxies can be used as a distance indicator [21]. This parameter measured in the I band with the ESO VLT, unit UT1 (ANTU) has been successfully used by Mei et al. [22] to retrieve the distance of the galaxy IC 4296 in Abell 3565. They found $49 \pm 4 \mathrm{Mpc}$. Another example is the determination of globular cluster (hereafter denoted GC) ages and metallicities using $(V-K)-(V-I)$ color diagrams. If the color index is measured with sufficient accuracy, some characteristics of the globular cluster evolution can be revealed such as a bimodality in population and/or in chemical composition [23]. These programs require precise calibrations of the telescope and detector setup with the different filters including flat-field imaging and sky background measurement.

Following a suggestion made by ESO, an experimental program was initiated

- to calculate the individual intensities and wavelengths of the $\mathrm{OH}$ ro-vibrational lines and

- to measure the intensity of the $\mathrm{OH}$ emission and the amplitude of its variations throughout the night.

The first part of the program was published under the form of an atlas of the $\mathrm{OH}$ spectrum between 0.997 and $2.25 \mu \mathrm{m}$ [6]. The second part is the subject of the present paper. Its aim is to measure and analyse the intensity and variability of the sky background when observing in the near infrared. The observational equipment used to measure the sky background is described in Section 2. The data analysis sequence to retrieve the sky intensity signal is explained. An outline is given of the different steps to obtain a measurement of the sky background as a function of time. In Section 3, observational results obtained in Europe at mid-latitudes are presented. Observations performed in Peru at latitudes of $12^{\circ} \mathrm{S}$ and $17^{\circ} \mathrm{S}$ are presented and analysed in Section 4. The publication is illustrated with several video films accessible on the web site http://www.obs-besancon.fr/nirsky/ Enter username: nirsky and password: skynir.

The movies show the motion of the sky background in an accelerated way. Three different movements are clearly apparent: the relative motion of the celestial sphere, the motion of the stripes in the mesospheric emissive layer and trails due to airplanes, satellites and meteors.

\section{Instrumentation and data reduction}

\subsection{Instrumentation}

The observation equipment set up for this program is mainly composed of a $512 \times$ 512 camera equipped with a Thomson CCD whose pixels are square, $19 \times 19 \mu^{2}$. 
An electromechanical shutter is used to precisely set the exposure time, generally comprised between 1 and $4 \mathrm{~min}$ at night-time. The $\mathrm{CCD}$ operates at $-40^{\circ} \mathrm{C}$ which reduces the noise level to $4 \mathrm{e}^{-} / \mathrm{pixel} / \mathrm{s}$. The well depth of a pixel is $290,000 \mathrm{e}^{-}$which allows using a 16 bit A-D converter.

The camera is fixed on a computer driven alt-azimuthal platform which can be pointed to any point of the upper sky hemisphere. Flat-field short time exposures are taken at dusk or at dawn when the signals from the stars become weak as compared to the level of the "blue" sky. During such sessions, in order to properly correct for the perceptible vignetting, the camera is pointed around the zenith. It is sequentially rotated by $90^{\circ}$ between exposures and, during exposures, moved by hand in an erratic way to avoid any star image in the flat-field picture. A number of $\sim 30$ (only $\sim 20$ at low latitude) flat-field images are then averaged. Several bias exposures provide the offset level. A number of $\sim 30$ dark images taken with the same exposure time as the sky images are averaged to produce the dark picture that is subtracted from the sky images.

In order to keep the exposure time as short as possible, a fast lens is used. Initially, a $28 \mathrm{~mm} \mathrm{f} / 2$ Nikon lens was mounted on the camera body. The field of view was $19^{\circ} \times 19^{\circ}$. Later, a new front plate for the camera with C-mount was designed. This camera modification made it possible to use an Angénieux $15 \mathrm{~mm}$

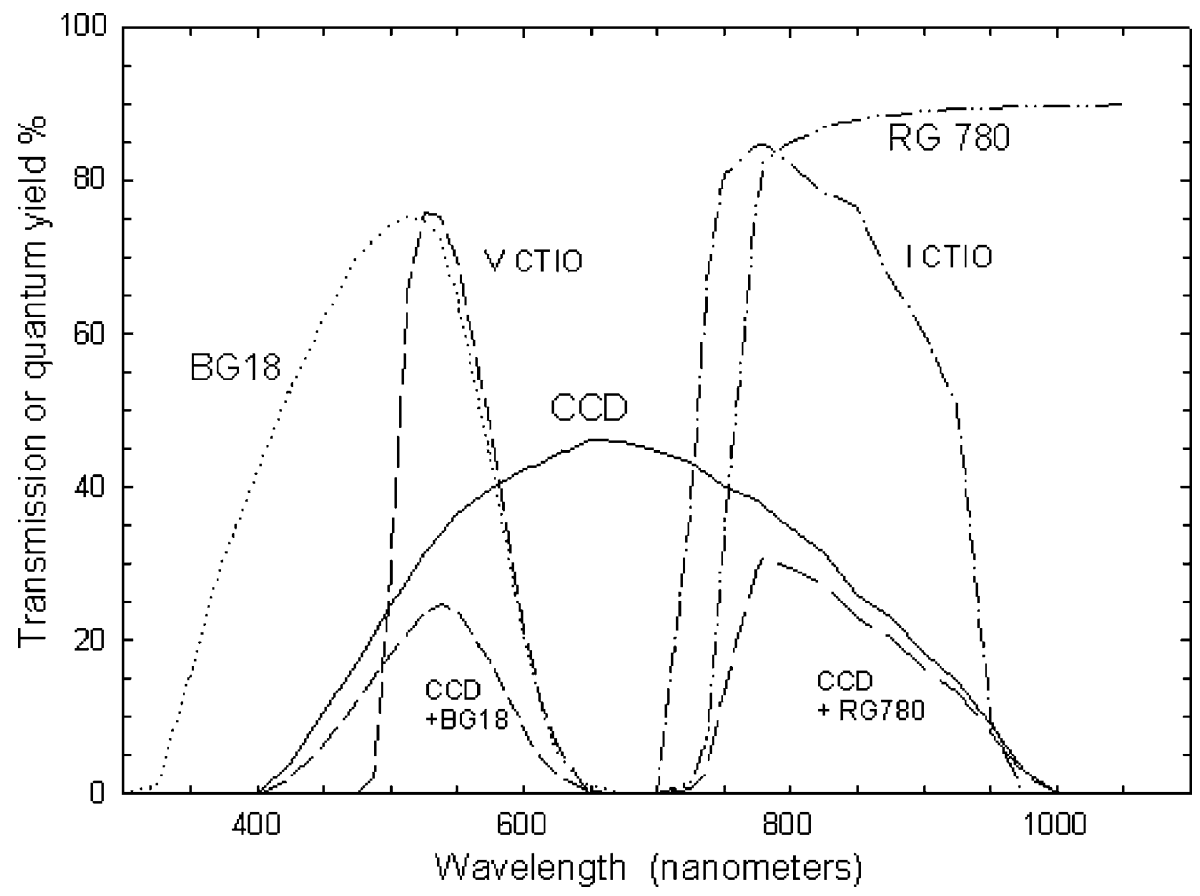

Fig. 3 Transmission curves of the visible BG 18/2 mm and NIR RG 780/2 mm filters. The quantum efficiency of the CCD and the resulting bandpass of the camera are also plotted. The transmission of the V and I filters used at CTIO [19] are shown for comparison with our camera bandpasses 
f/1.3 lens which provided a $36^{\circ} \times 36^{\circ}$ field of view. The actual focal length in the near-IR was measured and found equal to $15.6 \mathrm{~mm}$.

An RG 780/2 mm filter is placed in front of the lens. It is opaque in the visible and transmits radiation in the near-infrared at $\lambda>780 \mathrm{~nm}$. In the wavelength range between $780 \mathrm{~nm}$ and the cut-off of silicon at $1,020 \mathrm{~nm}$, the atmospheric emission is mainly composed of ro-vibrational bands of the $\Delta \nu=4$ sequence of $\mathrm{OH}$ denoted 9-5, $8-4,7-3,6-2,5-1$ and $4-0$. The near-IR filter can be removed and replaced by a visible BG39 or BG18 filter. The transmittance curves of the filters are presented in Fig. 3 together with the spectral quantum yield of the CCD retina. The resulting spectral bandwidth at half-maximum of the CCD camera plus the RG780 filter: 754 $900 \mathrm{~nm}, \Delta \lambda=146 \mathrm{~nm}$, may be compared with the bandwidth of the I filter used in the Denis survey [16]: $746-878 \mathrm{~nm}, \Delta \lambda=132 \mathrm{~nm}$ and the I interference filter used at CTIO [24]: 726-930 nm, $\Delta \lambda=204 \mathrm{~nm}$. As the bandpass of our instrument is very close to the I filter bandpass, we shall use the apparent magnitude $m_{\mathrm{I}}$ of stars in the field to calibrate the magnitude of the sky background emission. The $\mathrm{OH}$ spectrum in the 0.997-2.25 $\mu \mathrm{m}$ range (Fig. 4) was computed by Rousselot et al. [6] and compared with a spectrum taken with the Isaac spectrometer installed at the first unit telescope of the ESO-VLT. It is composed of ro-vibronic bands where each band contains several tens of lines.

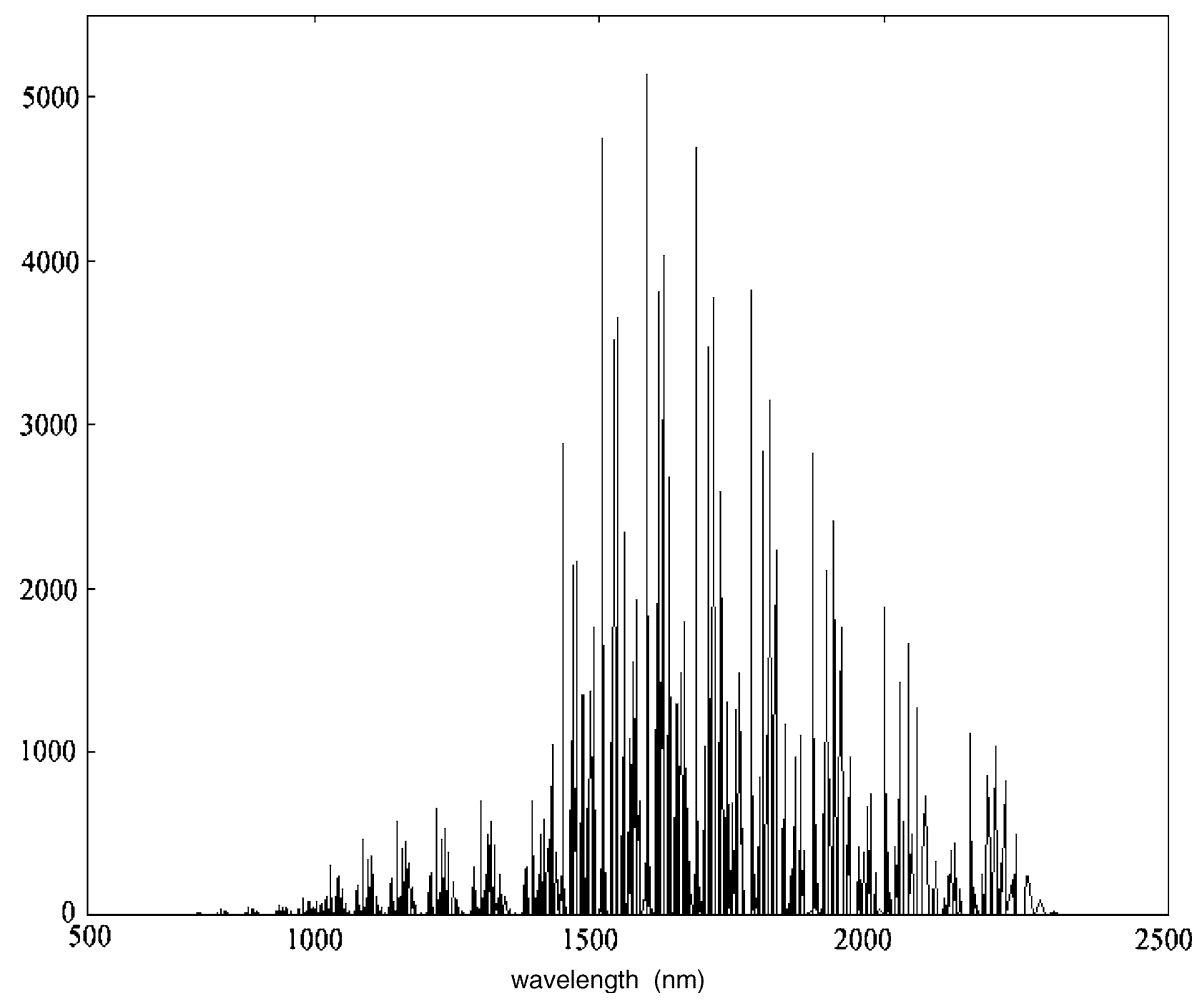

Fig. 4 General synthetic spectrum of the night-sky $\mathrm{OH}$ emission 


\subsection{Data reduction}

The first step in data reduction is to correct the image for flat-field and dark signals. The raw image is processed and improved in using Eq. (1):

$$
\text { Processed_image }=\frac{\text { Raw_image }- \text { Dark_image }}{\text { Flat_field_image }- \text { Bias_image }}
$$

The processed image is used to measure the intensity of a star and of the sky background in its vicinity. The second step is to correct for the Van Rhijn effect (Fig. 5). The effect is an increase of the atmospheric intensity due to an off-zenith observation, as the effective thickness of the atmospheric layer has increased. The intensification factor, called Van Rhijn factor is given by Eq. (2):

$$
V\left(h_{e m}, \theta\right)=\frac{1}{\sqrt{1-\frac{R^{2} \sin ^{2} \theta}{\left(R+h_{e m}\right)^{2}}}}
$$

In this expression, $h_{\mathrm{em}}$ is the altitude of the emissive layer and $R$ the Earth radius. When $\theta<\sim 70^{\circ}$, the expression of the Van Rhijn factor simplifies to: $V\left(h_{\mathrm{em}}, \theta\right) \approx \sec \theta$. For a purpose of comparison of the results obtained at different zenith angles, the sky background due to the mesospheric emissive layer is

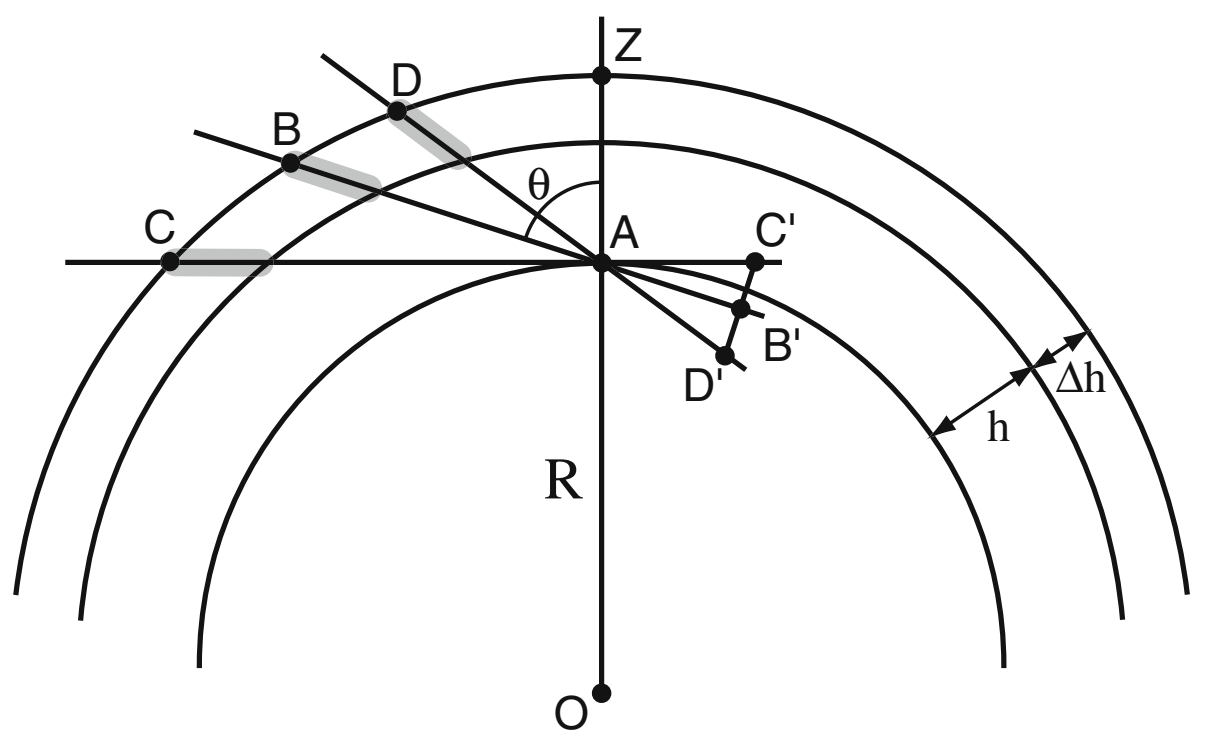

Fig. 5 Imaging the sky at slant angles through the atmosphere. The CCD camera is represented as a pinhole instrument. The contribution of the emissive layer increases with increasing zenith angle $\theta$ (Van Rhijn effect) 
expressed as reduced to zenith. The data measured at a given zenith distance will be divided by $V\left(h_{e m}, \theta\right)$ or multiplied by $\cos \theta$ if $\theta<\sim 70^{\circ}$ [25].

A correction for the air mass variation during the observing period is applied to the star intensity value. The value of the sky background is expressed in units of $I_{\text {mag20 }} / \operatorname{arcsec}^{2}$. After a process of flat-fielding and dark current subtraction, a $n$ by $n$ pixel box surrounding a field star is summed to give a value in ADU (analog to digital unit), $N_{1}$. An equivalent $n$ by $n$ box, located a small distance away with no stars in it, is also summed to give a value in $\mathrm{ADU}, N_{2}$. The stellar photon flux should therefore be $N_{1}-N_{2}$ in ADU, assuming the noise to be the same in each $n$ by $n$ box on the sky. The difference $N_{3}=N_{1}-N_{2}$ is referred to the star of apparent magnitude $m_{3}$ without background. Let us call $m^{\prime}$ the magnitude per $\operatorname{arcsec}^{2}$ of a fictitious extended object of magnitude $m_{3}$ that would be uniformly displayed over a $n^{2}$ pixel square. The relation between $m^{\prime}$ and $m_{3}$ is given by eq. (3):

$$
m^{\prime}=m_{3}+5 \log n+5 \log \frac{a}{f}+5 \log 206,265
$$

In this expression, $a$ is the size of the pixel, $f$ the focal length of the lens and 206,265 the number of arcseconds in a radian. Next, we calculate the ratio $\rho=$ $10^{0.4\left(20-m^{\prime}\right)}$. Finally, the ADU figure for one unit of $I_{\operatorname{mag} 20} / \operatorname{arcsec}^{2}$ that is used to measure the atmospheric emission in the zenith is $N_{20}=\frac{N_{3}}{\rho \cos \theta}$. In the following sections, it will be shown that the sky background in the I band measured during our campaigns at mid and low latitudes is comprised between 1 and $3.5 I_{\operatorname{mag} 20} / \operatorname{arcsec}^{2}$.

Fig. 6 Image of the sky taken in the near-IR at Observatoire de Haute Provence on October 19, 1998 at 20:10 UT. A series of stripes extend over the $19^{\circ} \times 19^{\circ}$ field of view. The outline of the $152-\mathrm{cm}$ telescope dome is visible in the left lower part of the image

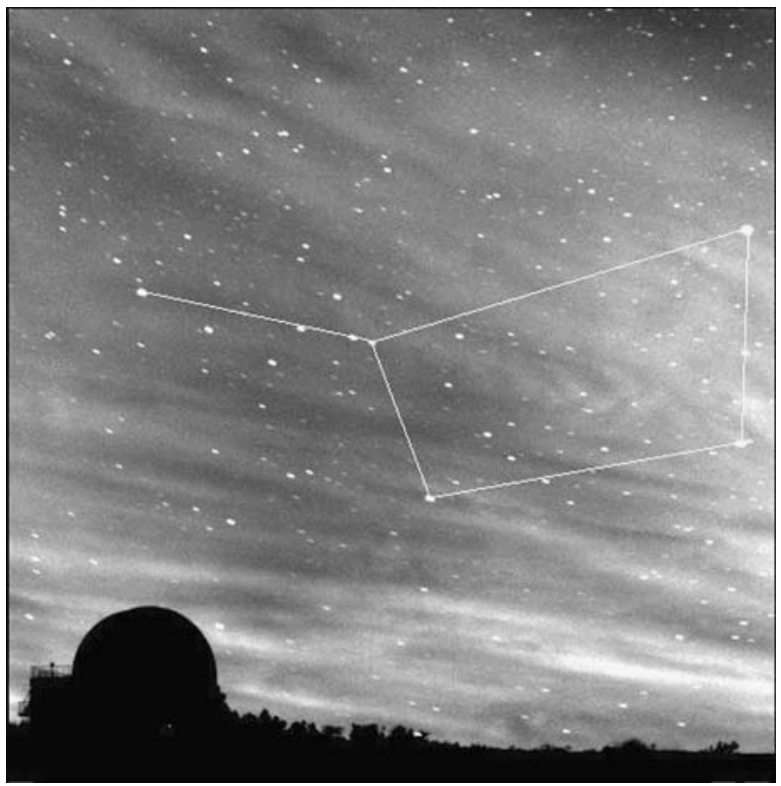




\section{Observations at mid-latitudes}

An image of the sky taken in the I bandpass of the camera at Observatoire de Haute Provence $\left(43^{\circ} 56^{\prime} \mathrm{N}, 5^{\circ} 43^{\prime} \mathrm{E}\right.$, hereafter called OHP) on October 19, 1998, 20:10 UT is presented in Fig. 6. A whole series of stripes regularly spaced extends over the $19^{\circ} \times 19^{\circ}$ field of view (Fig. 7). Five stars of the Big Dipper are easily identified. Dubhe and Merak are aligned vertically close to the right side of the image. Megrez is located close to the center. Phecda, below is the fourth vertex of the Big Dipper quadrilateral. Alioth is on the left of Megrez, above the $1.52 \mathrm{~m}$ telescope dome. A comparison of the intensity of the sky with the signals of Alioth $\left(m_{\mathrm{I}}=1.82\right)$ and Megrez $\left(m_{\mathrm{I}}=3.25\right)$ shows that the intensity of the $\mathrm{OH}$ emission in the $\mathrm{I}$ band is equivalent to that which would be produced by a number of stars of magnitude 10 per square degree of the sky comprised between 1,300 and 5,200. This is equivalent to one star of magnitude $1.48 \pm 0.8$ per square degree. This figure is comparable to the illumination produced in the visible by the moon at $65^{\circ}$ phase angle.

A more precise evaluation of the near-IR sky background was conducted at OHP during the night $19-20$ October 1998. Sequential images of a $19^{\circ} \times 19^{\circ}$ field were taken with an exposure time of $1 \mathrm{~min}$ from 21:45 to 5:45 UT. A series of 12 consecutive images taken at time intervals of $4 \mathrm{~min}$ from 04:50 to 05:34 UT at the

Fig. 7 Same image as in Fig. 6, but projected in a different way. The perspective effect has been inverted and the image shown is a satellite type view, i.e. a vertical downward view of the emissive layer. The structures seen in Fig. 6 appear as periodic stripes comparable to the surface of the sea

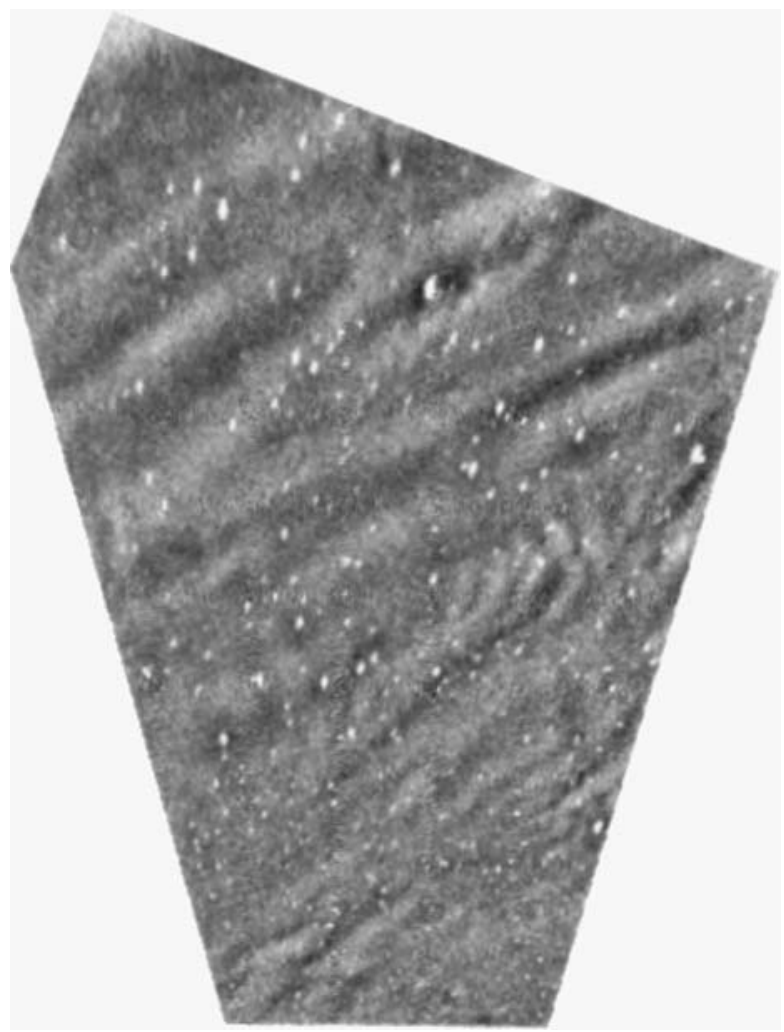


end of the night is presented in Fig. 8. This set of photographs shows that an intensity wave depicted by the varying emission intensity propagates through the field of view. In order to measure the equivalent magnitude of the emission, the intensities of three stars, called A (TYC 4650-3036-1; $\alpha_{2000}=22$ h 54 min $25 \mathrm{~s}$; $\left.\delta_{2000}=84^{\circ} 20^{\prime} 46^{\prime \prime}\right)$, B (TYC 4632-2067-1; $\alpha_{2000}=10$ h $08 \mathrm{~min} 34 \mathrm{~s} ; \delta_{2000}=83^{\circ} 55^{\prime}$ $\left.06^{\prime \prime}\right)$ and C (TYC 4629-37-1; $\alpha_{2000}=04$ h $\left.42 \min 46 \mathrm{~s} ; \delta_{2000}=89^{\circ} 37^{\prime} 48^{\prime \prime}\right)$ are monitored as a function of time during the night. Stars A and B shown in Fig. 9 are nearly symmetric with respect to the pole. Figure 10 presents the evolution as a function of time of the intensities of stars A, B and C plus the sky background as measured in adding up the pixel values on a $6 \times 6$ pixel square area. We also measure the intensity of the sky background in a $6 \times 6$ pixel area devoid of stars and located close to the selected stars. Star C close to the pole is very weak compared to A and B and, consequently, the intensity in curve $(C)$ is mainly due to the sky background. In order to give a quantitative estimate of the background, we present in Fig. 11 three curves showing the evolution with time of the star B + background, of the sky background and of the star B. The star intensity slightly increases because its air mass diminishes as its zenith angle decreases from $51^{\circ} 25^{\prime}$ to $40^{\circ} 55^{\prime}$ throughout the night. The apparent magnitude of star B is now used to calibrate the intensity scale of the sky background in the NIR at 754-900 nm. The intensity is expressed in terms of $I_{\text {mag20 }}$ per $(\operatorname{arcsec})^{2}$. The sky background intensity is expressed as reduced to
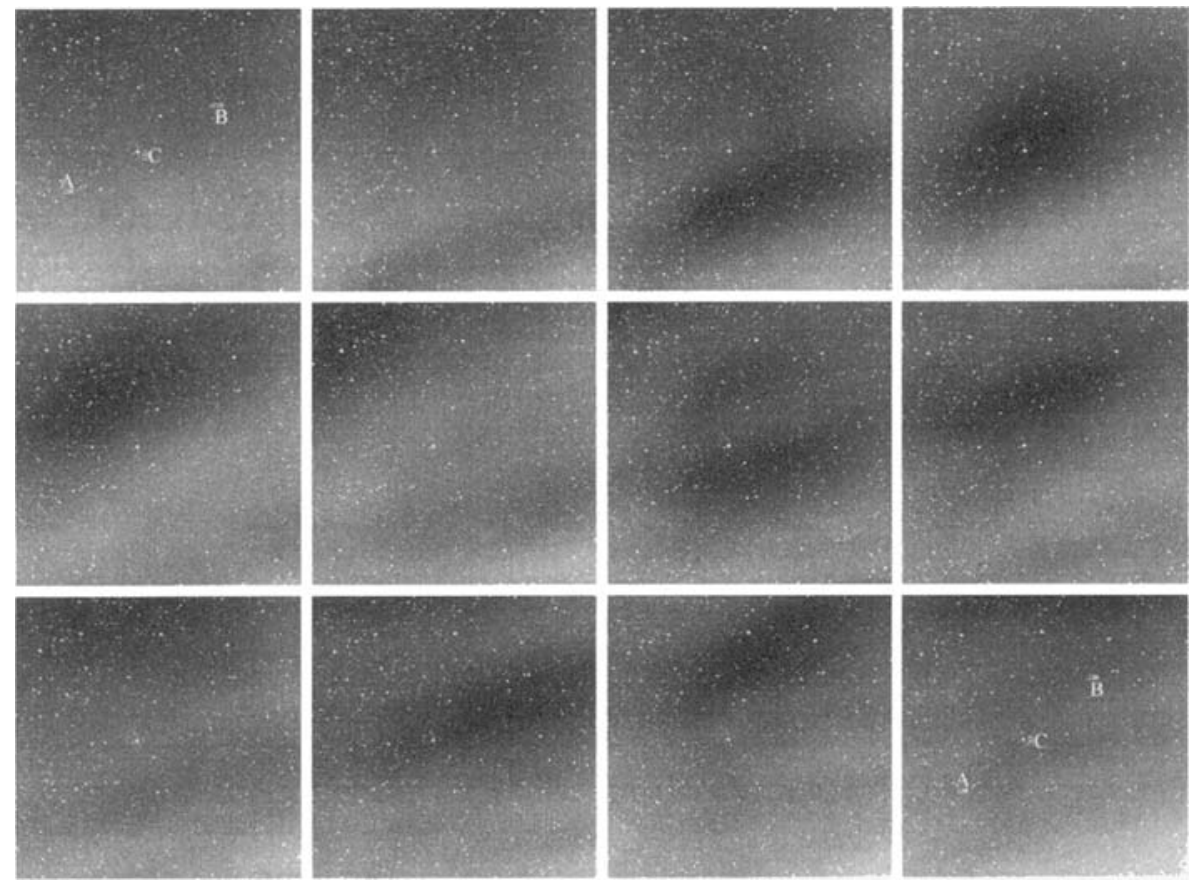

Fig. 8 Set of 12 consecutive images taken at time intervals of 4 min from 04:50 to 05:34 UT at the end of the night of $19-20$ October 1998 . The observation site is OHP. The $19^{\circ} \times 19^{\circ}$ field of view is centered on Polaris. Two successive waves characterized by a dark tilted stripe across the image moving upward are clearly visible in this set of photographs 
Fig. 9 Positions of the 3 circumpolar stars called $A$ (TYC 4650-3036-1), $B$ (TYC 46322067-1) and $C$ (TYC 4629-37-1) inside the $19^{\circ} \times 19^{\circ}$ field of view of the camera. Stars $A$ and $B$ are nearly symmetric with respect to the pole
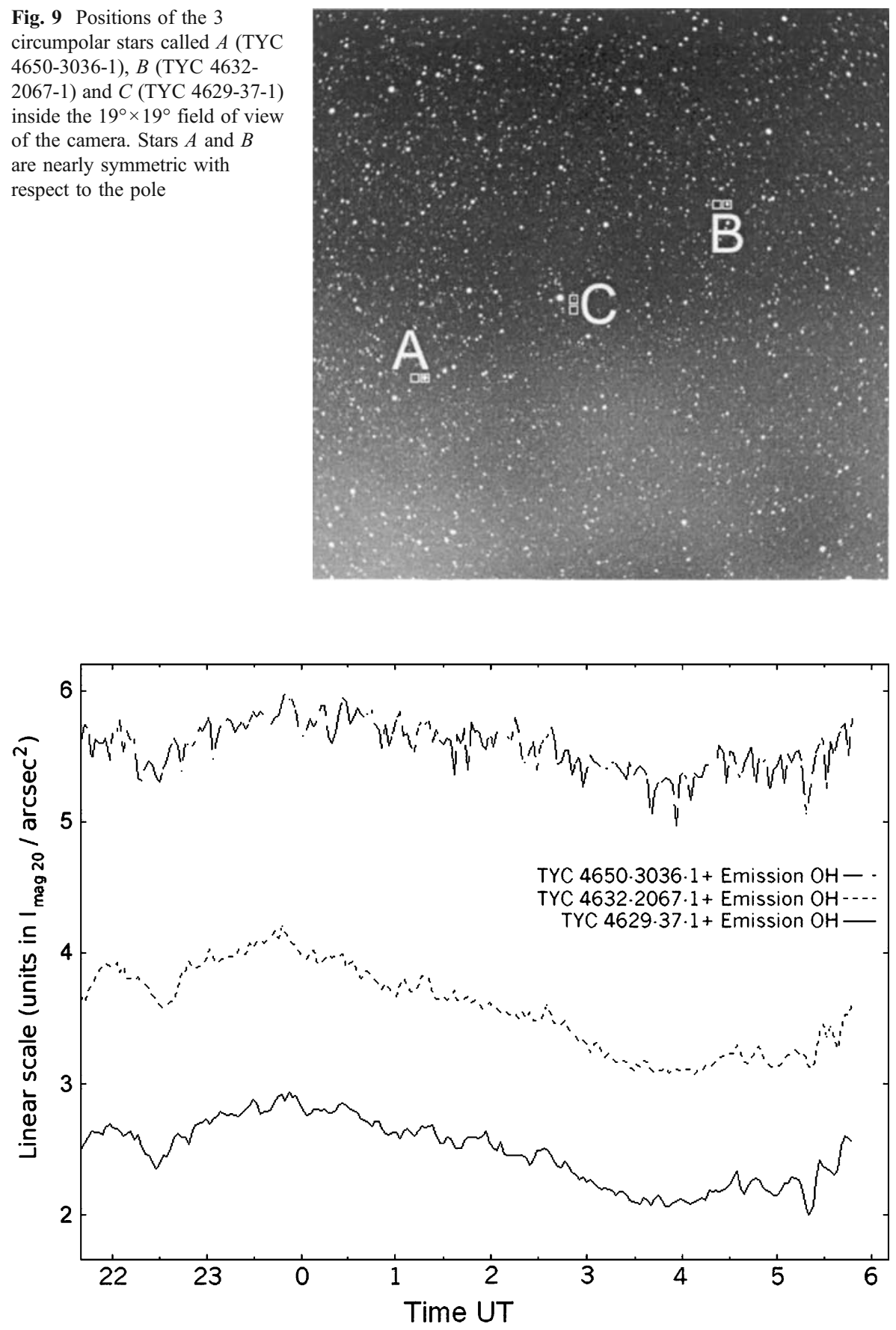

Fig. 10 Evolution, as a function of time from 21:50 to 05:40 UT during the night 19-20 October 1998, of the intensities of the stars plus background measured in a square area of 36 pixels $\left(196 \operatorname{arcmin}^{2}\right)$. Upper curve star A (TYC 4650-3036-1). Middle curve star B (TYC 4632-2067-1). Lower curve star C (TYC 4629-37-1). The intensities are expressed with a linear scale in units of $I_{\operatorname{mag} 20} / \operatorname{arcsec}^{2}$ in the I band. They are reduced to zenith at zero airmass 


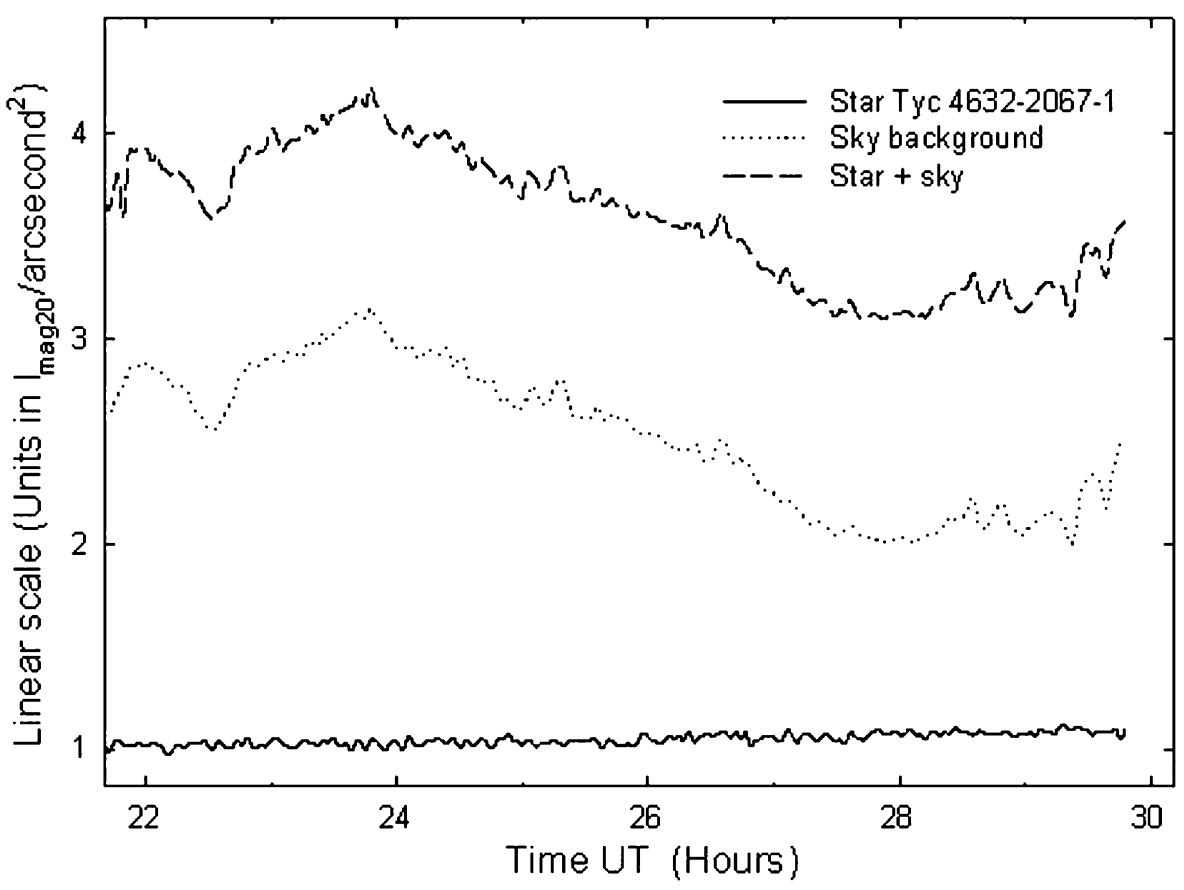

Fig. 11 Evolution, as a function of time from 21:50 to 05:40 UT during the night 19-20 October 1998, of the intensities of the star B+background, of the sky background close to star B and of the star without background (difference between the two previous curves)

zenith in taking into account the Van Rhijn factor $V\left(h_{\mathrm{em}}, \theta\right)$. The final result is presented in Fig. 12 where the intensity scale is linear. A fast Fourier analysis of the intensity curves of Fig. 12 was performed in order to retrieve the periods of the variations of the sky background. Three main periods are apparent at 16.5, 8.9 and $5.7 \mathrm{~min}$ (Fig. 13). The short period, $5.7 \mathrm{~min}$, is typical of buoyancy oscillations [12, p. 32]. The major period of $16.5 \mathrm{~min}$ can be detected in examining the twelve successive images in Fig. 8 covering a time interval of $44 \mathrm{~min}$. This short period corresponds to the propagation of waves having an horizontal wavelength comprised between 20 and $50 \mathrm{~km}$. The resulting intensity fluctuation is about 10 to $15 \%$ of the background intensity. A slower variation of greater amplitude is visible in Fig. 12. From 22:00 till midnight, the sky background increases by $18 \%$. A secondary minimum occurs at 22:40 during $30 \mathrm{~min}$. After midnight, the intensity decreases down to reach at 04:00 UT $68 \%$ of its maximum value at 23:30. During the last part of the night, it increases again.

The evolution of the sky background during the night shows two components:

- A slowly varying component mainly due to the evolution of the atmospheric chemical composition and to density variation involved by harmonic periods of diurnal tides. The amplitude of these variations can be as high as $50 \%$.

- Fluctuations with periods of 5.7 to $16.5 \mathrm{~min}$ and their harmonics. These fluctuations have an amplitude of $15 \%$. They are due to the propagation of atmospheric gravity waves. 


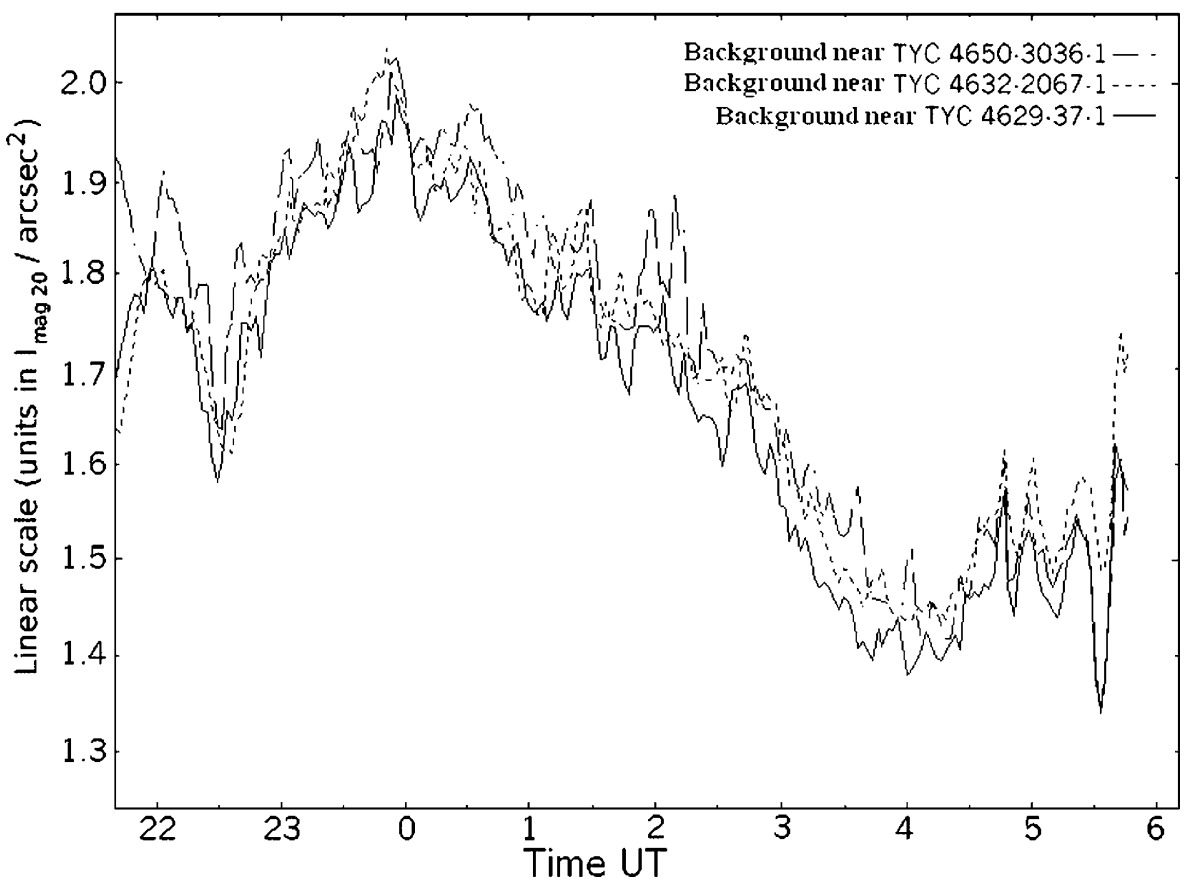

Fig. 12 Evolution, as a function of time during the night of 19-20 October 1998, of the sky background intensity expressed with a linear intensity scale and units of $I_{\operatorname{mag} 20} / \operatorname{arcsec}^{2}$ in the NIR I band

Other observations conducted at the observatory of Château-Renard in July 2000 show comparable variations of the sky background intensity [26].

\section{Observations at low latitudes}

In October 2005 and July 2006, a program of stereoscopic observations of the mesospheric emissive layer was conducted in Peru. The instrumental layout was the same as the one performed in September 2000 at the Pic du Midi and ChâteauRenard observatories [27]. Observations were made simultaneously at two sites distant of $645 \mathrm{~km}$. A first site was located at Cerro Cosmos $\left(12^{\circ} 09^{\prime} 08.2^{\prime \prime} \mathrm{S}, 75^{\circ} 33^{\prime}\right.$ 49.3" W, altitude: 4,630 m) close to Huancayo. In this place, a solar observatory was built in 1976. It was active until 1988 when it could not any longer be operated. A second site was located at Cerro Verde Tellolo (16 33' 17.6" S, 71 $39^{\prime} 59.4^{\prime \prime} \mathrm{W}$, altitude $2,330 \mathrm{~m}$ ), close to Arequipa. The stereoscopic procedure consisted in observing a common area, located at half-distance of both sites, in the mesospheric emissive layer, in order to retrieve the altitude of this layer. As a result, the azimuths for the optical axes were $318^{\circ}$ for Cerro Cosmos and $138^{\circ}$ for Cerro Verde. We now present two types of observations. The first one is a series of measurements of the sky background at Cerro Cosmos covering $8.4 \mathrm{~h}$ during the night of 24-25 July 2006. The second is a series of measurements of the sky background close to the Small Magellan Cloud performed at Cerro Verde during 30 to 90 min on 23-24, 25-26 and 28-29 July 2006. 


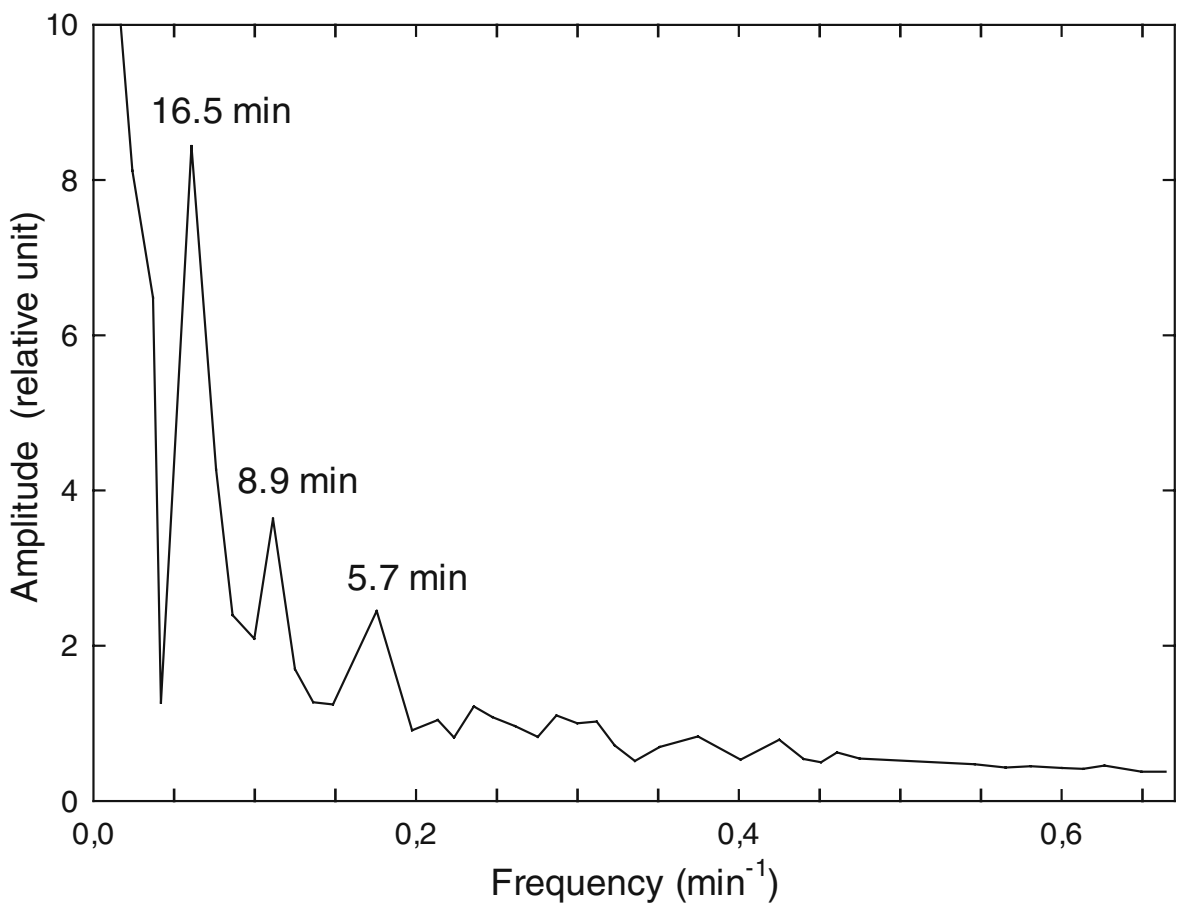

Fig. 13 Frequency spectrum of the NIR sky background intensity during the night of 19-20 October 1998. Three main periods are apparent at $16.5,8.9$ and $5.7 \mathrm{~min}$

4.1 Measurement of the near-IR sky background at Cerro Cosmos during the night of 24-25 July 2006

The center of the field of view was set at azimuth $318^{\circ}$ and angular altitude $18^{\circ} 40^{\prime}$. The intensity curve of Fig. 14 shows the evolution with time of the emission of the sky background in the I band. The values are reduced to zenith in multiplying the

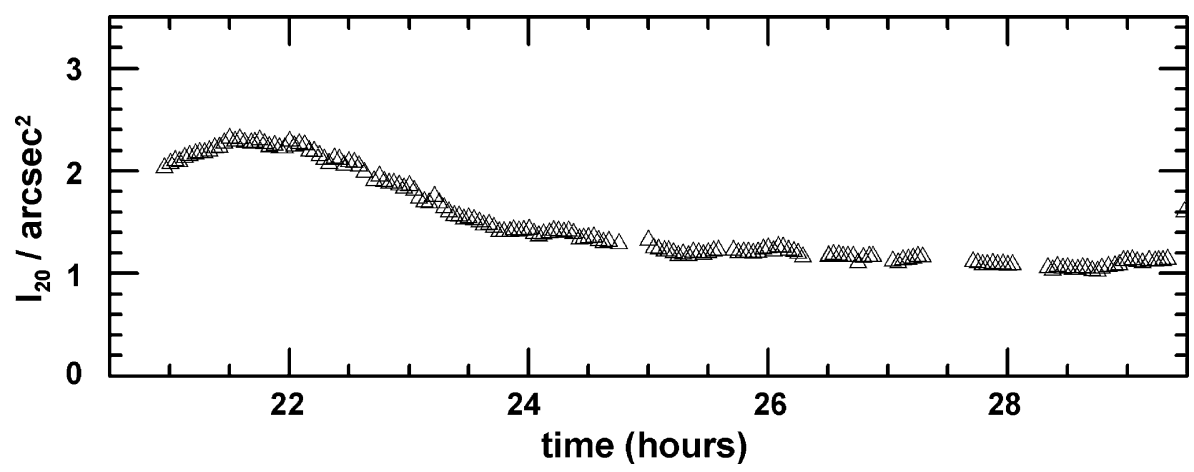

Fig. 14 Evolution as a function of time of the sky background recorded at Cerro Cosmos during the night of 24-25 July 2006. The time scale is UT $-5 \mathrm{~h}$. The geographic coordinates of the observed emissive zone in the mesosphere are $12^{\circ} 48^{\prime} \mathrm{S}$ and $75^{\circ} 23^{\prime} \mathrm{W}$. The intensity is expressed with a linear scale and units of $I_{\text {mag20 }} / \operatorname{arcsec}^{2}$ in the NIR I band 
measured intensity by $\cos \theta$ where $\theta$ is the zenith angle of the measured square area. At the beginning of the night, the intensity is $\sim 2 \times I_{\text {mag20 }} / \operatorname{arcsec}^{2}$. Then it increases to a maximum of $2.4 \times I_{\operatorname{mag} 20} / \operatorname{arcsec}^{2}$ at 21:50. During the following $7 \mathrm{~h}$, the intensity decreases to the value of $(1.1) \times I_{\operatorname{mag} 20} / \operatorname{arcsec}^{2}$ at the end of the night.

The conversion of the intensity expressed in analog-digital units (ADU) to solid angle magnitude units is made in the following way. A star of apparent magnitude $m_{\mathrm{I}}=3$ to 4 is chosen close to the center of the field of view. The intensity sum in a $5 \times 5$ pixel area containing the star image is recorded as a function of time until the star reaches the upper limit of the field of view. On each image, the sky background intensity is measured in a $5 \times 5$ pixel area devoid of star and located close to the chosen star on the same horizontal line. The difference between both measurements provides the star intensity which is corrected for the air mass extinction. The apparent magnitudes of stars in the I band is taken in the Aladin data base [33]. They are listed in Table 1. The variation of the intensity as a function of time is shown in six successive panels referred as (a) to (f) in Fig. 15, a-f.

The variation of the sky background during the night of 24-25 July 2006 is as high as $130 \%$. In terms of apparent magnitude, the intensity is comprised between a maximum of $m_{1}=19.1 / \operatorname{arcsec}^{2}$ and a minimum of $m_{2}=20.0 / \operatorname{arcsec}^{2}$. As the decrease occurs mainly during $3 \mathrm{~h}$, the sky variation of $m_{\mathrm{I}}$ is about $0.3 / \mathrm{h}$. Two major processes influence the variation of the atmospheric near-IR emission: the evolution with time of the chemical stationary state and the dynamic perturbations propagating

Table 1 List of the stars used to measure the intensity of the sky background

\begin{tabular}{|c|c|c|c|c|c|c|c|c|c|c|}
\hline Denomination & $\begin{array}{l}\text { RA } \\
(\mathrm{J} 2000)\end{array}$ & $\begin{array}{l}\text { DEC } \\
(\mathrm{J} 2000)\end{array}$ & $\begin{array}{l}\text { Sp. } \\
\text { Type }\end{array}$ & $m_{\mathrm{B}}$ & $m_{\mathrm{V}}$ & $m_{\mathrm{R}}$ & $m_{\mathrm{I}}$ & $m_{\mathrm{J}}$ & $m_{\mathrm{H}}$ & Present use \\
\hline $\begin{array}{l}\text { TYC 4629- } \\
37-1\end{array}$ & $04: 42: 46$ & $89: 37: 48$ & $\mathrm{~K} 2$ & 10.801 & 9.163 & 8.280 & 7.3 & 6.264 & 5.582 & Star C-OHP \\
\hline $\begin{array}{l}\text { TYC 4632- } \\
2067-1\end{array}$ & $10: 08: 34$ & $83: 55: 06$ & K0 & 7.920 & 6.334 & & 5.32 & 3.238 & 2.459 & Star B-OHP \\
\hline Megrez & $12: 15: 26$ & $57: 01: 57$ & A3 V & 3.421 & 3.314 & 3.25 & 3.284 & 3.11 & & \\
\hline Alioth & $12: 54: 02$ & $55: 57: 35$ & A0 & 1.813 & 1.764 & 1.79 & 1.82 & 1.72 & & \\
\hline $\begin{array}{l}\text { TYC 4650- } \\
3036-1\end{array}$ & $22: 54: 25$ & $84: 20: 46$ & K4 III & 6.568 & 4.853 & 3.63 & 2.90 & 2.382 & & Star A-OHP \\
\hline $\begin{array}{l}\text { TYC 8035- } \\
687-1\end{array}$ & 01:04:24 & $-47: 56: 25$ & G6 & 8.482 & 6.863 & 5.980 & 4.7 & 3.657 & & $\begin{array}{l}\text { Star c) } \\
\text { Cosmos }\end{array}$ \\
\hline Chi Phenicis & 02:01:42 & $-44: 42: 49$ & K5 III & 6.63 & 5.14 & 3.92 & 3.03 & 2.52 & & $\begin{array}{l}\text { Star d) } \\
\text { Cosmos }\end{array}$ \\
\hline $\begin{array}{l}\text { TYC 8067- } \\
2459-1\end{array}$ & $03: 45: 16$ & $-47: 21: 34$ & K1 III & 6.663 & 5.714 & 5.120 & 4.4 & 4.179 & 3.662 & $\begin{array}{l}\text { Star e) } \\
\text { Cosmos }\end{array}$ \\
\hline Zeta Pictoris & $05: 19: 22$ & $-50: 36: 21$ & F7 III & 6.05 & 5.498 & & 4.908 & 4.731 & & $\begin{array}{l}\text { Star f) } \\
\text { Cosmos }\end{array}$ \\
\hline $\begin{array}{l}\text { TYC 8441- } \\
198-1\end{array}$ & $22: 09: 58$ & $-48: 06: 28$ & K2 & 7.856 & 6.435 & 5.560 & 4.4 & 3.817 & 3.088 & $\begin{array}{l}\text { Star a) } \\
\text { Cosmos }\end{array}$ \\
\hline $\begin{array}{l}\text { TYC 8456- } \\
967-1\end{array}$ & $23: 57: 51$ & $-45: 29: 32$ & K3 III & 4.804 & 4.727 & 4.690 & 4.58 & 4.530 & 4.450 & $\begin{array}{l}\text { Star b) } \\
\text { Cosmos }\end{array}$ \\
\hline Theta Octans & $00: 01: 36$ & $-77: 03: 57$ & K3 III & 6.430 & 4.928 & 3.81 & 3.14 & & & Magellan \\
\hline
\end{tabular}

The magnitudes are retrieved from the JP11 Ducati [34] or the NOMAD (Zacharias [35]) catalogs accessible in the Aladin Data Base [33] at http://vizier.u-strasbg.fr/viz-bin/VizieR. When the I magnitude was not quoted, it was interpolated using the V, R and J values. TYC 8456-967 is of special interest because its magnitude exhibits a small variation between 0.4 and $1.2 \mu \mathrm{m}$ 
a

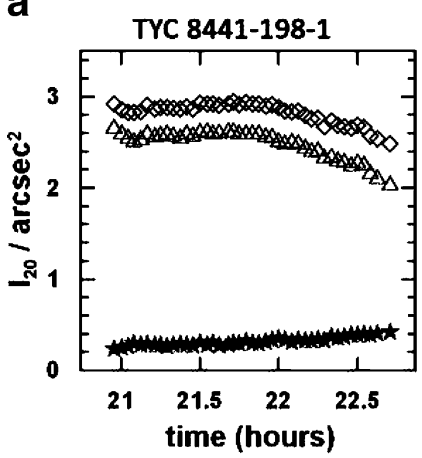

b

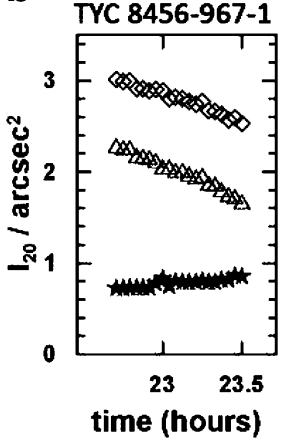

C

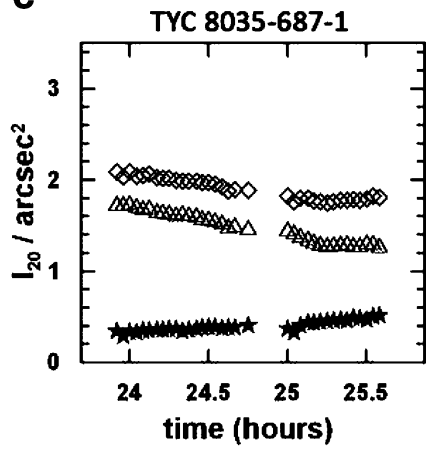

e

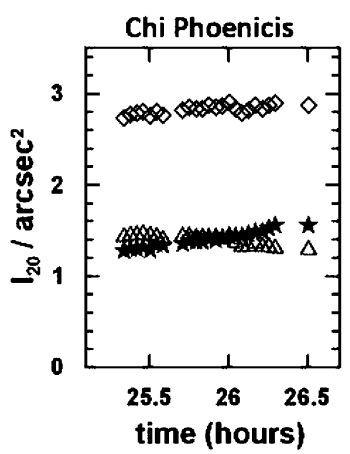

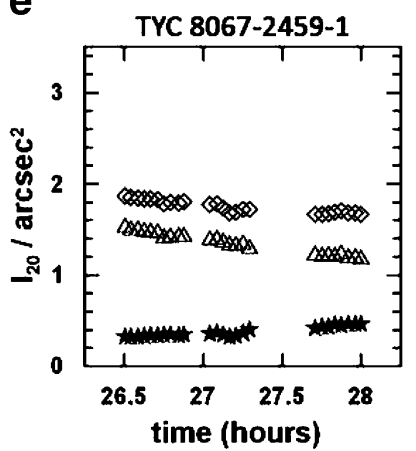

f

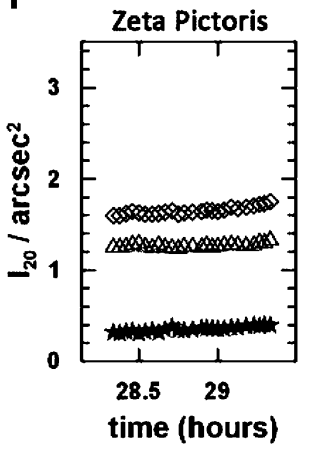

Fig. 15. a, b, c, d, e, f Set of six panels showing the intensities of six stars measured successively at Cerro Cosmos during the night of 24-25 July 2006. The time scale is UT-5 h. Each panel depicts for a specific star three intensity curves versus time: star+background, background only and star without background (difference between the two previous curves). The time intervals in the different panels are comprised between 1 and $2 \mathrm{~h}$. The apparent motion of each star is nearly vertical upward because the latitude is low and the azimuth is $318^{\circ}$. The list of the stars is given in Table 1

through the emissive layer. The $\mathrm{OH}^{*}$ radicals are produced in the reaction of atomic hydrogen with ozone [3]. The profile of hydrogen increases with altitude, whereas the profile of ozone strongly decreases. The $\mathrm{OH}^{*}$ emission is mainly a function of the density product $[\mathrm{H}]\left[\mathrm{O}_{3}\right]$ whose maximum is located around $87 \mathrm{~km}$. Chemical models [28] show that the $\mathrm{OH}$ emissive layer is $\sim 7 \mathrm{~km}$ thick within the $82-92 \mathrm{~km}$ altitude range They predict that, as soon as the upper atmosphere is no longer illuminated by the Sun, the ozone concentration should rapidly increase, while the atomic hydrogen that is produced during the day by water vapor dissociation should decrease. The resulting evolution of the $\mathrm{OH}^{*}$ with time throughout the night, if only chemical processes are considered, should be first an increase, followed by a maximum and a slow decrease during the remaining $2 / 3$ of the night. The variations observed during the night 24-25 July 2006 closely follow this chemical scheme. In the present case, dynamical processes do not dominate the chemical processes.

\subsection{Sky background close to the Small Magellan Cloud}

Several time periods were secured for measuring the near-IR sky background in the vicinity of the Small Magellan cloud. The results presented hereafter are related to 
a

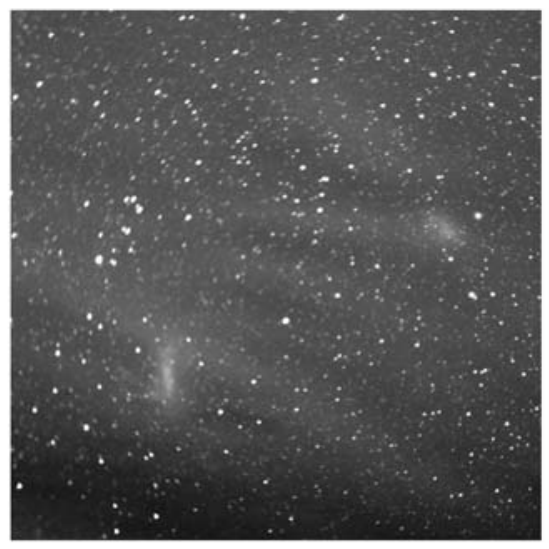

C

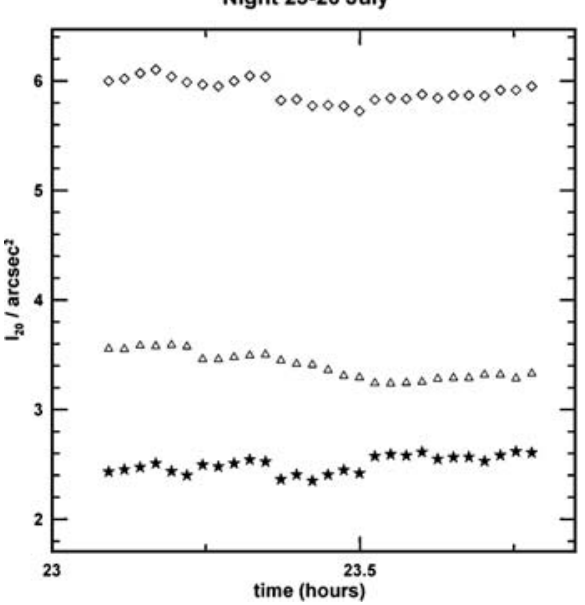

b

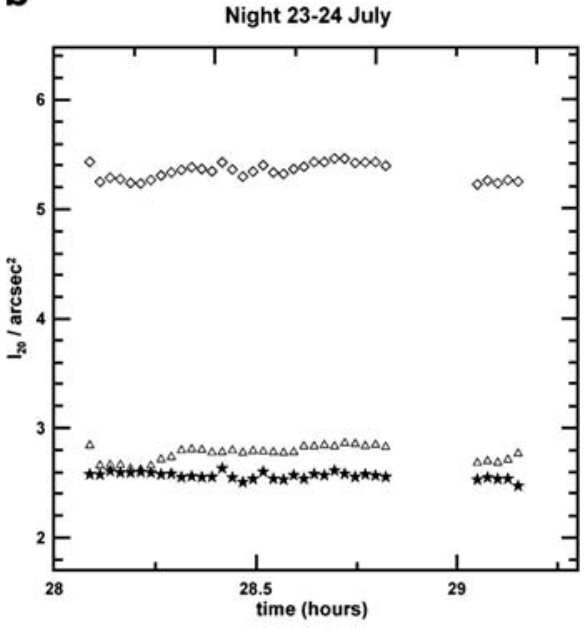

d

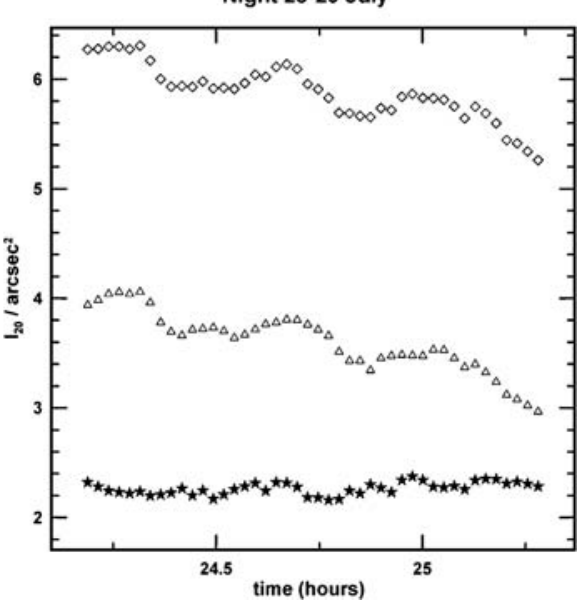

Fig. 16. a, b, c, d a) Image of the sky taken in the near-IR at Cerro Verde at the end of the night of 23-24 July 2006. The time scale is UT $-5 \mathrm{~h}$. The Small Magellan Cloud is visible on the right side. The Large Magellan Cloud is seen in the left lower quadrant. The south pole is located close to the right lower corner. A series of four stripes due to the mesospheric emission appears in the lower half part of the image. The field of view is $36^{\circ} \times$ $36^{\circ}$. Set of 3 intensity measurement sequences b) during one hour at the end of the night of 23-24 July, c) during one hour in the middle of the night of 25-26 July and d) during one hour in the middle of the night of 27-28 July 2006. Each panel depicts for Theta Octans three intensity curves versus time: star+background, background only and star without background (difference between the two previous curves). The durations of the time sequences in the different panels are comprised between 1 and $2 \mathrm{~h}$

three observing sequences during the nights 23-24, 25-26 and 28-29 July 2006. The procedure is the same as exposed in Section 4.1 and in Fig. 16, panels a to f. An image of the field of view including both Magellan clouds is presented in Fig. 16a. The reference star for the three sequences is Theta Octans (SAO 258207), $m_{\mathrm{I}}=3.14$. In the three b, c, d panels of Fig. 16, three curves are presented. The upper curve shows the star+sky intensity. The intermediate curve shows the sky background and 
the lower one is for the star alone without background. It may be seen that the sky background is not constant. It is comprised between 2.7 and 4.1 units of $I_{\text {mag20 }} /$ $\operatorname{arcsec}^{2}$. The panel Fig. 16d shows intensity fluctuations having an amplitude of 0.5 unit of $I_{\operatorname{mag} 20} / \operatorname{arcsec}^{2}$ and a period of $17 \mathrm{~min}$. Such a period lies within the period values range calculated by Faivre et al. [27] using Fourier analysis.

\section{Discussion}

The quantitative importance of the mesospheric emission, of its spatial and temporal variations in the near-IR was pointed out by Maihara et al. [29] who measured the $\mathrm{OH}$ spectrum in the J 1.14-1.34 $\mu \mathrm{m}$ and $\mathrm{H} 1.50-1.80 \mu \mathrm{m}$ bands. Their conclusion was that, if observations can be performed in very narrow spectral regions devoid of $\mathrm{OH}$ lines, then the sky background emission could be reduced to $\sim 2 \%$ of its value including the lines. This spectral constraint is however difficult to satisfy when designing a focalplane instrument because the widest intervals free of lines are just $\Delta \lambda=30 \mathrm{~nm}$ at $1,370-1,400 \mathrm{~nm}$ between the $9-6$ and $2-0$ band and $\Delta \lambda=80 \mathrm{~nm}$ between the $6-4$ and 7-5 bands. The intervals between individual spectral lines are narrower than $10 \mathrm{~nm}$.

Cuby et al. [30] measured the sky background with the Infrared Spectrometer and Array Camera (ISAAC) installed at the VLT Unit1-Antu. They found values of 16.5 and $14.4 \mathrm{mag} / \mathrm{arcsec}^{2}$ respectively in the $\mathrm{J}$ and $\mathrm{H}$ bands. The measured continuum at $1.67 \mu \mathrm{m}$ is four times higher than the figure reported by Maihara et al. [29] but 13 to 50 times lower than the $\mathrm{OH}$ emission in the $\mathrm{H}$ band.

Another comparison with the sky background intensity reported here in Sections 3 and 4 can be made in considering the values mentioned by Mei et al. [22] who quote an average $m_{\text {sky,I }}=19.09 \mathrm{mag} / \mathrm{arcsec}^{2}$ on June 19,1994 and $m_{\text {sky,I }}=18.26 \mathrm{mag} /$ $\operatorname{arcsec}^{2}$ on July 24,1994 . These values are 2.3 and 4.97 in linear units of $I_{\text {mag20 }} /$ $\operatorname{arcsec}^{2}$, which are comparable with our measurements comprised between 1 and 4.1 $I_{\text {mag20 }} / \operatorname{arcsec}^{2}$. The amplitude of the variation of the sky background due to the mesospheric near-IR emission expressed in magnitude is $\Delta m=0.44$ for the variation throughout the night. This decrease is interpreted as reflecting the diminution of the density product of ozone and atomic hydrogen in the mesosphere. During nighttime, photolysis processes are inefficient and do not dissociate water molecules. There is no production of atomic hydrogen whereas ozone is stable after a brief increase following sunset. Consequently, the sky background variation observed during the night of 24-25 July at Cerro Cosmos appears as due to chemical processes in the mesosphere. Periodic fluctuations of smaller amplitude $\Delta m=0.15$ were observed at OHP (Fig. 12). Their period was equal to several tens of minutes. They are interpreted as a result of the propagation of gravity waves.

Such variations, that have amplitudes comprised between $15 \%\left(\Delta m_{\mathrm{I}}=0.15\right)$ for the rapid ones and $100 \%\left(\Delta m_{\mathrm{I}}=0.75\right)$ for the slow ones, are large compared to the precision required on the $V-I$ color index to deduce pertinent information about a possible bimodality when observing a population of globular clusters in distant galaxies. In the last 15 years, Zepf and Ashman [31] and other groups showed that the histogram giving the number of GCs in color bins of $\Delta(V-I)=0.1$ between 0.76 and 1.4 present two maxima at $V-I=0.95 \pm 0.02$ and $1.14 \pm 0.02$. This discovery was called "color bimodality" in extragalactic GC population. It was analyzed as a 
partition in two subpopulations: those with a blue color index being metal poor GCs and those with a red color index being metal rich GCs [32]. In addition, GCs are considered as good tracers of the star formation spheroids (early-type galaxies, spiral bulges and halos). Consequently, the NIR magnitudes of GCs appear as fundamental parameters in the study of galaxy formation, complementary to specific studies of galaxies.

In a recent paper related to colour-colour diagrams and extragalactic ages, Salaris and Cassisi [23] show that, if photometric parameters are in error by $\Delta m=0.1 \mathrm{mag}$, the bimodality expressed in population age differences cannot be detected if this difference is less than 8 Gyr. In this example, most observations were performed from space with the HST/WFPC2 instrument. In present days, such programs are also conducted with ground-based telescopes. They are concerned with the additional contribution due to the atmospheric emission which cannot by any means be suppressed.

\section{Conclusion}

Emission lines produced by the $\mathrm{OH}$ radical dominate the near infrared spectra. They strongly restrict astronomical observations of faint objects in the 0.6-2.6 $\mu \mathrm{m}$ spectral range because they are spatially and temporally variable. As a result, spectroscopic and/or astrometric observations in the range of the I, Y, J, H, K filters require that a non-negligible fraction of the allowed telescope time be used for nodding the telescope out of the field in order to measure the sky background in a region without stars close to the object of interest. Following a suggestion made by ESO, we analysed photometric measurements of the $\mathrm{OH}$ nightglow from observations previously conducted at OHP during the night of 19-20 October 1998. The intensity of three circumpolar stars at polar distances $<7^{\circ}$ was recorded during $7 \mathrm{~h} 50 \mathrm{~min}$. The time variations, due to the sky background, were expressed in units of $I_{\text {mag20 }} / \operatorname{arcsec}^{2}$ suitable for extended sources. Two components were depicted in the intensity curve:

1. a slowly varying component that reflects the evolution with time of the chemical composition of the mesospheric layer and also the influence of harmonic components of the atmospheric tide having periods of 4 and $6 \mathrm{~h}$. The amplitude of this type of variation is as high as $50 \%$ between 2.1 and $3.0 I_{\operatorname{mag} 20} / \operatorname{arcsec}^{2}$.

2. periodic fluctuations with periods of 5-20 $\mathrm{min}$ and their harmonics. These fluctuations have an amplitude of $\sim 15 \%$.

In October 2005 and July 2006, a program of stereoscopic observations of the mesospheric $\mathrm{OH}$ layer was conducted at two sites in Peru: Cerro Cosmos $\left(12^{\circ} 09^{\prime} \mathrm{S}\right.$, $75^{\circ} 34^{\prime} \mathrm{W}$, altitude 4,630 $\left.\mathrm{m}\right)$ and Cerro Verde Tellolo $\left(16^{\circ} 33^{\prime} \mathrm{S}, 71^{\circ} 40^{\prime} \mathrm{W}\right.$, altitude $2,330 \mathrm{~m})$. The sky background in the I band was monitored during a complete 8.5-h night on 24-25 July and during two partial nights on 25-26 and 27-28 July 2006. The sky background variation during the $8.5 \mathrm{~h}$ observation period on 24-25 July 2006 is very comparable to that observed at OHP in October 1998. In terms of apparent magnitude in the I band, the sky background was comprised between $m_{1}=19.1 / \operatorname{arcsec}^{2}$ and $m_{2}=20.0 / \operatorname{arcsec}^{2}$. During the 3 -h decrease phase from $22 \mathrm{~h} 00(03 \mathrm{~h} \mathrm{UT})$ to $25 \mathrm{~h}$ local $(06 \mathrm{~h} \mathrm{UT})$, the variation of the sky magnitude was about $0.3 / \mathrm{h}$. The observations at Cerro Verde Tellolo show that the sky background intensity was comprised between 2.7 and 4.1 units of $I_{\text {mag20 }} /$ 
$\operatorname{arcsec}^{2}$. On 28-29 July, between $24.2 \mathrm{~h}$ and $25.6 \mathrm{~h}$, intensity oscillations were recorded. Their temporal period was $17 \mathrm{~min}$ and their intensity amplitude was 0.5 unit of $I_{\operatorname{mag} 20} / \operatorname{arcsec}^{2}$.

As a conclusion, we would suggest that a NIR camera similar to the one described in Section 2 might record images of the sky in a $36^{\circ} \times 36^{\circ}$ field of view centered on the direction of the observed object. This camera would measure the magnitude fluctuations of the atmospheric emission in order to precisely subtract the sky background contribution. The interest of having a fairly wide field of view is to record the evolution of the $\mathrm{OH}$ emission in the direction of the telescope field of view. The camera platform could easily be driven in the equatorial mode in order to monitor the sky background in the vicinity of the object of interest during the observing session.

Acknowledgements Sweetest thanks are due to Marie-Jeanne for constant help during this scientific program.

Open Access This article is distributed under the terms of the Creative Commons Attribution Noncommercial License which permits any noncommercial use, distribution, and reproduction in any medium, provided the original author(s) and source are credited.

\section{References}

1. Meinel, A.B.: OH emission bands in the spectrum of the night sky. Astrophys. J. 111, 555-564 (1950)

2. Herzberg, G.: The atmospheres of the planets. J. R. Astron. Soc. Can. 45, 100-123 (1951)

3. Anlauf, K.G., McDonald, R.G., Polanyi, J.C.: Infrared chemiluminescence from $\mathrm{H}+\mathrm{O}_{3}$ at low pressure. Chem. Phys. Lett. 1, 619-622 (1968)

4. Osterbock, D.E., Fulbright, J.P., Martel, A.R., Keane, M.J., Trager, S.C., Basri, G.: Night-sky high resolution spectral atlas of $\mathrm{OH}$ and $\mathrm{O}_{2}$ emission lines for echelle spectrograph wavelength calibration. Publ. Astron. Soc. Pac. 108, 277-308 (1996)

5. Osterbrock, D.E., Fulbright, J.P., Bida, T.A.: Night-sky high resolution spectral atlas of OH emission lines for echelle spectrograph wavelength calibration. II. Publ. Astron. Soc. Pac. 109, 614-627 (1997)

6. Rousselot, P., Lidman, C., Cuby, J., Moreels, G., Monnet, G.: Night-sky spectral atlas of OH emission lines in the near-infrared. Astron. Astrophys. 354, 1134-1150 (2000)

7. Peterson, A.W., Kieffaber, L.M.: Infrared photography of $\mathrm{OH}$ airglow structures. Nature 242, 321-322 (1973)

8. Moreels, G., Hersé, M.: Photographic evidence of waves around the $85 \mathrm{~km} \mathrm{level.} \mathrm{Planet.} \mathrm{Space} \mathrm{Sci.}$ 52, 265-273 (1977)

9. Clairemidi, J., Hersé, M., Moreels, G.: Bi-dimensional observation of waves near the mesopause at auroral latitudes. Planet. Space Sci. 33, (9), 1013-1022 (1985)

10. Taylor, M.J., Gardner, L.C., Pendleton, W.R.: Long period waves signatures in mesospheric $\mathrm{OH}$ Meinel $(6,2)$ band intensity and rotational temperature at midlatitudes. Adv. Space Res. 27, (6-7), 1171-1179 (2001)

11. Tarasick, D.W., Hines, C.O.: The observable effects of gravity waves on airglow emissions. Planet. Space Sci. 38, (9), 1105-1119 (1990)

12. Andrews, D.G.: An Introduction to Atmospheric Physics, p. 229. Cambridge University Press, Cambridge (2000)

13. Coulman, C.E., Vernin, J., Fuchs, A.: Optical seeing mechanism of formation of thin turbulent laminae in the atmosphere. Appl. Opt. 34, 5461-5474 (1995)

14. Vernin, J.: Mechanism of formation of optical turbulence. In: Vernin, J., Benkhaldoun, Z., MuñozTuñón, C. (eds.) Astronomical Site Evaluation in the Visible and Radio Range. ASP Conference Series vol. 266, pp. 2-27 (2002) 
15. Avila, R., Ziad, A., Borgnino, J., Martin, F., Agabi, A., Tokovinin, A.: Theoretical spatiotemporal 9 analysis of angle of arrival induced by atmospheric turbulence as observed with the grating scale monitor experiment. J. Opt. Soc. Am. A. 14, (11), 3070-3082 (1997)

16. Schultheis, M., Ganesh, S., Glass, I.S., Omont, A., Ortiz, R., Simon, G., van Loon, J.Th., Alard, C., Blommaert, J.A.D.L., Borsenberger, J., Fouqué, P., Habing, H.J.: DENIS and ISOGAL properties of variable star candidates in the galactic bulge. Astron. Astrophys. 362, 215-222 (2000)

17. Skrutskie, M. F., Schneider, S.F., Stiening, R. and 15 co-authors: The two micron all sky survey (2MASS), overview and status. In: P. Garzón et al. (eds.) The Impact of Large Scale Near-IR Sky Surveys, 25, Kluwer (1997)

18. Cabrera-Lavers, A., Garzón, F., Hammersley, P.L., Vicente, B., González-Fernández, C.: TCS-CAIN: a deep multi-colour NIR survey of the Galactic plane. Astron. Astrophys. 453, DOI 10.1051/00044631:20042200, 371-385 (2006)

19. Dalton, G., Caldwell, M., Ward, K., 20 co-authors: The Vista IR camera. SPIE 5492, 988-997 (2004)

20. Emerson, J.P., Sutherland, W.J., McPherson, A.M., Craig, S.C., Dalton, G.B., Ward, A.K.: The visible infrared survey telescope for astronomy. The ESO Messenger 117, 27-32 (2004)

21. Mei, S., Quinn, P.J., Silva, D.R.: Anomalous surface brightness fluctuations in NGC 4489. Astron. Astrophys. 366, 54-61 (2001). DOI 10.1051/0004-6361:20000080

22. Mei, S., Silva, D.R., Quinn, P.J.: VLT Deep I-band surface brightness fluctuations of IC 4296. Astron. Astrophys. 361, 68-72 (2000)

23. Salaris, M., Cassisi, S.: Colour-colour diagrams and extragalactic globular cluster ages. Astron. Astrophys. 461, 493-508 (2007). DOI 10.1051/0004-6361:20066042

24. Suntzeff, N.B., Walker, A.R.: The CTIO prime-focus charge-coupled device: system characteristics from 1982-1998. Publ. Astron. Soc. Pacific. 108, 265-270 (1996)

25. Pautet, D., Moreels, G.: Ground-based satellite-type images of the upper atmosphere emissive layer. Appl. Opt. 41, (5), 823-831 (2002)

26. Pautet, D., Moreels, G., Rousselot, P., Reylé, C., Clairemidi, J.: Observing at Oukaïmeden through the extended wave system of the upper atmosphere emissive layer. In: Vernin, J., Benkhaldoun, Z., Muñoz-Tuñó, C. (eds.) Astronomical site evaluation in the visible and radio range. ASP Conference Series vol. 266, pp. 28-35 (2002)

27. Faivre, M., Moreels, G., Pautet, D., Clairemidi, J.: Fourier analysis of a virtual satellite view of the NIR atmospheric emission over Europe. Adv. Space Res. 32, (5), 843-848 (2003)

28. Allen, M., Yung, Y.L., Waters, J.W.: Vertical transport and photochemistry in the terrestrial mesosphere and lower thermospher (50-120 km) J. Geophys. Res. 86, 3617-3627 (1981)

29. Maihara, T., Iwamuro, F., Yamashita, T., Hall, D.N.B., Cowie, L.L., Tokunaga, A.T., Pickles, A.: Observations of the airglow emission. Publ. Astron. Soc. Pacif. 105, 940-944 (1993)

30. Cuby, J.G., Lidman, C., Moutou, C.: ISAAC: 18 months of paranal science operations. The ESO Messenger 101, 2-8 (2000)

31. Zepf, S.E., Ashman, K.: Globular cluster systems formed in galaxy mergers. MNRAS 264, (3), 611618 (1993)

32. Brodie, J.P., Strader, J.: Extragalactic globular clusters and galaxy formation. Ann. Rev. Astron. Astrophys. 44, 193-267 (2006)

33. Aladin Data base, Centre de Données Stellaires, Observatoire de Strasbourg

34. Ducati, J.R.: Catalogue of stellar photometry in Johnson's 11 color system. CDS/ADC Collection of electronic catalogues II/237

35. Zacharias, N., Monet, D.G., Levine, S.E., Urba, S.E., Gaume, R., Wyckoff, G.L.: NOMAD Catalog, VizieR on-line data catalog I/297 (2005) 